\title{
Análisis de la calidad de vida del paciente en la etapa prediálisis
}

\author{
María del Carmen Ruiz Parrado, Mercedes Gómez Pozo, María Crespo Garrido, Carmen Tierno Tendero, \\ Rodolfo Crespo Montero
}

Unidad de Gestión Clínica de Nefrología. Hospital Universitario Reina Sofía de Córdoba. España

\section{Resumen}

Objetivos: El objetivo del presente estudio fue analizar la Calidad de Vida Relacionada con la Salud de los pacientes con Enfermedad Renal Crónica Avanzada en estadio 5.

Pacientes y Método: Se estudiaron a 32 pacientes (41\% mujeres), con una edad media de $67.3 \pm 12.2$ años, con Enfermedad Renal Crónica Avanzada en estadio 5 y un filtrado glomerular $<15 \mathrm{mil} / \mathrm{min}$. Se realizó un estudio observacional descriptivo y transversal, en el Servicio de Nefrología del Hospital Reina Sofía de Córdoba. Para valorar la Calidad de Vida Relacionada con la Salud se utilizó el cuestionario KDQOL-SF. Otra variable estudiada fue la comorbilidad asociada.

Resultados: Las dimensiones más afectadas fueron: la situación laboral, la función sexual, el rol físico, la salud general y la función física. Por el contrario, las mejor conservadas fueron: la función cognitiva, la calidad de las relaciones sociales, el apoyo social, la función social y el bienestar emocional. No se encontraron diferencias significativas en ninguna de las dimensiones respecto al sexo.

Se encontró una asociación entre mayor edad y peor función sexual, función física, rol físico y vitalidad. También se correlacionó estadísticamente la comorbilidad con función física y vitalidad; y con la edad.

Correspondencia:

María del Carmen Ruiz Parrado

Urbanización Bahía Doncella, $n^{\circ} 2$, Bajo

29680 Estepona. Málaga

E-mail:n32rupam@uco.es
Conclusión: Los pacientes con Enfermedad Renal Crónica Avanzada en estadio 5 tienen disminuida su Calidad de Vida Relacionada con la Salud en comparación con la población sana de referencia; siendo la situación laboral, la función sexual, función física y salud general, las dimensiones más afectadas. La edad y la comorbilidad se asociaron a peor calidad de vida.

PALABRAS CLAVE: ERCA; calidad de vida relacionada con la salud; prediálisis; diálisis.

\section{Analysis of the quality of life of the patient in the predialysis stage}

\section{Abstract}

Aim: The objective of the present study has been analysed the Health-Related Quality of Life of patients who had Advanced Chronic Kidney Disease.

Patients and method: Thirty-two patients were studied ( $41 \%$ women), with an average age of $67.3 \pm 12.2$ years old, with Stage 5 Advanced Chronic Kidney Disease and a glomerular filtration rate $<15 \mathrm{mil} / \mathrm{min}$. A descriptive and cross-sectional observational study was performed at the Nephrology Department of the Reina Sofía Hospital of Córdoba. The KDQOL-SF questionnaire was used to assess the Quality of Life Related to Health. Associated comorbidity was also studied.

Results: The most affected dimensions were: the work situation, sexual function, physical role, general health and physical function. On the contrary, the best preserved ones were: cognitive function, quality of social relations, social support, social function and emotional well-being. No significant differences were found in any of the aspects with respect to gender. 
A significant relationship was found between age and sexual function, physical function, physical role and vitality. Statistically correlated comorbidity with physical function and vitality were also statistically correlated; Also with age.

Conclusion: Patients with advanced stage 5 chronic kidney disease have decreased their health-related quality of life compared to the healthy reference population; Being the work situation, sexual function, physical function and general health, the most affected dimensions. Age and comorbidity were associated with poorer quality of life.

KEYWORDS: ACKD; health related quality of life; pre-dialysis; dialysis.

\section{Introducción}

Según los resultados obtenidos sobre la prevalencia de Enfermedad Renal Crónica (ERC) en España por el Estudio Epidemiológico de la Insuficiencia Renal Crónica en España (EPIRCE), se estableció que la prevalencia global de ERC en estadios 3-5 era del 6,8\%, siendo los factores de riesgo cardiovasculares los que presentaban una considerable prevalencia, además de la dislipemia, la obesidad, el tabaquismo activo, la hipertensión y la diabetes. Por otro lado, se observó que otros factores predictores independientes de ERC fueron la edad, la obesidad y la hipertensión diagnosticada previamente. Esta prevalencia estaba ligeramente elevada, independientemente del estadio, sobre todo en personas de mayor edad ${ }^{1}$.

La Enfermedad Renal Crónica Avanzada (ERCA) es una alteración que viene ocasionada por la presencia de un filtrado glomerular $(F G$ ) inferior a $15 \mathrm{ml} / \mathrm{min}$, como consecuencia de una lesión estructural o funcional del riñón. La ERCA, coincide con el último estadio (estadio 5) de la Enfermedad Renal Crónica (ERC) y, a su vez, con la etapa de prediálisis ${ }^{2,3}$. En este último estadio, es cuando es necesario comenzar una Terapia Renal Sustitutiva (TRS).

En España, según el Registro de Diálisis y Trasplantes de 2015, de la Sociedad Española de Nefrología, la prevalencia de pacientes en TRS era el siguiente: el $52,5 \%$ de los pacientes estaban con trasplante renal funcionante, el $41,9 \%$ estaban en hemodiálisis (HD) y el $5,5 \%$ estaban en diálisis peritoneal. La mayoría de los pacientes que presentan esta enfermedad tienen una edad comprendida entre los 65 y los 74 años, siendo la técnica de diálisis más prevalente, la HD4 .

Estamos pues, ante una enfermedad cuya prevalencia aumenta con la edad y que, en un momento, dado va a necesitar una TRS. Esta enfermedad, además de limitante físicamente, va a acarrear a la persona que la padece importantes alteraciones en todas las esferas de su vida, social, familiar, laboral, etcétera ${ }^{5}$; repercutiendo directamente en su calidad de vida, evaluada como Calidad de Vida Relacionada con la Salud (CVRS) 6 , y en la realización de las Actividades de la Vida Diaria $(\mathrm{AVD})^{7}$.

Se ha señalado, que además del deterioro de la función renal ocasionado por la ERCA, la disminución de la CVRS viene determinada por otras patologías asociadas y/o las complicaciones que origina la ERC ${ }^{5}$. Por este motivo, la ERC, debe ser controlada por los equipos multidisciplinares en las consultas de ERCA, porque se ha comprobado que un mejor control retrasa las complicaciones, y porque es muy importante, centrarse no sólo en las variables biológicas de la enfermedad sino también en otros parámetros como la calidad de vida $^{8}$. De este modo, una remisión precoz a las consultas de ERCA, acompañada de una buena labor llevada a cabo por el equipo multidisciplinar, podría suponer una mejora, tanto del nivel de la CVRS, como del afrontamiento de la enfermedad ${ }^{3,8}$.

Sin embargo, aunque la CVRS está muy estudiada en pacientes con ERCA que se encuentran en TRS, especialmente en diálisis ${ }^{9-11}$, no existe mucha bibliografía que analice esta variable en el paciente en la etapa previa a la entrada en TRS o estadio 5.

Por todo ello, dada la importancia que tiene la percepción de la CVRS en el paciente con ERCA, y la falta de estudios realizados sobre ésta en pacientes en la etapa de prediálisis, nos planteamos el siguiente trabajo, con el propósito de analizar la CVRS que tienen los pacientes en esta etapa.

Por consiguiente, el objetivo principal de este estudio ha sido analizar la CVRS de los pacientes con ERCA en la etapa de pre-diálisis, con los siguientes objetivos específicos:

- Identificar las dimensiones más afectadas en la CVRS en los pacientes en pre-diálisis.

- Identificar las variables más influyentes en la calidad de vida de estos pacientes. 


\section{Pacientes y Método}

\section{Ámbito de estudio}

El estudio tuvo lugar en la Unidad de Gestión Clínica (UGC) de Nefrología del Hospital Universitario Reina Sofía de Córdoba (HURS), en la consulta de Bajo Aclaramiento.

\section{Diseño}

Se realizó un estudio observacional descriptivo transversal.

\section{Población y muestra}

La población estudiada fueron los pacientes con ERCA en estadio 5 atendidos en la consulta de Bajo Aclaramiento de la UGC de Nefrología del HURS. De todos ellos, se dividió a los mismos en función del FG tomando como punto de corte un FG de $15 \mathrm{ml} / \mathrm{min}$, con objeto de seleccionar a aquellos pacientes que están más próximos a ser incluidos en TRS. Con un FG $<15 \mathrm{ml} /$ min, eran seguidos en consulta 60 pacientes en el momento del inicio del estudio.

Tras la identificación de estos pacientes por parte de los responsables de esta consulta, se iniciaron las entrevistas a todos aquellos pacientes que entre marzo y mediados de mayo acudieron a la misma y tras leer el consentimiento informado y la hoja informativa del estudio, aceptaron participar.

Finalmente se obtuvo una muestra de 32 pacientes.

\section{Variables del estudio}

Variable de resultado: CVRS

Otras variables: edad, sexo y comorbilidad.

\section{Instrumentos de medida}

Para evaluar la CVRS se utilizó el cuestionario validado KDQOL-SF, que es una adaptación del cuestionario SF-36 para pacientes con ERCA.

Para el análisis de la comorbilidad se utilizó el Índice de Comorbilidad de Charlson (ICC).

\section{Recogida de datos}

La recogida de datos se realizó entre los meses de marzo y mediados de mayo, coincidiendo con la consulta de Bajo Aclaramiento de la UGC Nefrología, en función de la cita programada en esta consulta. El cuestionario fue cumplimentado en el tiempo que estuvieron en consul- ta y la entrevista fue realizada por única investigadora con la colaboración de la enfermera de esta consulta, por lo que no fue necesario que el paciente dedicara tiempo extra para colaborar en el estudio. Algunos pacientes solicitaron cumplimentar el cuestionario en su domicilio, a lo que se accedió después de comprobar que entendían las preguntas del mismo.

En todo momento, se garantizó la confidencialidad de los datos recogidos.

\section{Aspectos éticos}

La información obtenida ha sido utilizada exclusivamente para los fines de esta investigación y es totalmente confidencial y tratada conforme a la Ley Orgánica 15/1999 de 13 diciembre, sobre "Protección de datos de carácter personal". Si en algún momento el paciente deseó retirarse del estudio, lo pudo hacer sin tener que dar ningún tipo de explicación. Se garantizó en todo momento el anonimato de las pacientes.

El estudio se inició después de recibir el informe favorable del Comité de Ética e Investigación del HURS.

\section{Análisis estadístico}

Para las variables cualitativas se utilizó una distribución de frecuencias, mientras que para las variables numéricas se usó la media \pm la desviación estándar de la media y en algunos casos la mediana. Para la comparación entre variables se utilizó la t de Student para medias independientes y la $U$ de Mann-Whitney según siguieran o no una distribución normal. También se realizaron correlaciones con el test de Pearson. Se aceptó significación estadística para $p<0.05$. El análisis estadístico se ha realizado en un paquete estadístico SSPS 15.0 para Windows.

\section{Resultados}

Se estudiaron 32 pacientes con una edad media de $67.3 \pm 12.2$ años. Respecto al sexo 19 eran hombres $(59 \%)$ y 13 mujeres $(41 \%)$, con un ICC de $5.81 \pm 2.76$. No se encontraron diferencias en el ICC entre hombres y mujeres.

Respecto a los resultados del KDQOL-SF, en la siguiente tabla están representados los valores de la Escala Específica para Enfermedades Renales (tabla 1).

En la tabla 2, se representan los valores obtenidos en las dimensiones del SF-36. 
Tabla 1. Valores de la Escala Específica para Enfermedades Renales (KDQOL-SF).

\begin{tabular}{|lcc|}
\hline $\begin{array}{l}\text { Escalas Específicas para } \\
\text { Enfermedades Renales }\end{array}$ & Media y DT & Mínimo-Máximo \\
\hline Listado de síntomas / problemas & $73.9 \pm 18.2$ & $20.45-100$ \\
\hline Efectos de la enfermedad renal & $66.3 \pm 20.8$ & $25-100$ \\
\hline Carga de la enfermedad renal & $52.7 \pm 25.3$ & $0-100$ \\
\hline Situación laboral & $32.8 \pm 35$ & $0-100$ \\
\hline Función cognitiva & $83.8 \pm 21.5$ & $20-100$ \\
\hline Calidad de las relaciones & $89.4 \pm 16.1$ & $40-100$ \\
sociales & $51.2 \pm 35.5$ & $0-100$ \\
\hline Función sexual & $68.7 \pm 19.6$ & $22.50-100$ \\
\hline Sueño & $85.4 \pm 23.1$ & $0-100$ \\
\hline Apoyo social & & \\
\hline
\end{tabular}

DT: Desviación típica

Tabla 2. Valores de las dimensiones del SF-36 (KDQOL-SF).

\begin{tabular}{lcc}
\hline Ítems del SF-36 & Media y DT & Mínimo-Máximo \\
\hline Función física & $52.6 \pm 29.7$ & $5-100$ \\
\hline Rol físico & $34.4 \pm 41.5$ & $0-100$ \\
\hline Dolor & $61.4 \pm 29.4$ & $10-100$ \\
\hline Salud general & $39.3 \pm 16.9$ & $5-70$ \\
\hline Bienestar emocional & $70 \pm 19.8$ & $20-100$ \\
Rol emocional & $67.7 \pm 43.6$ & $0-100$ \\
Función social & $82.4 \pm 22.2$ & $25-100$ \\
Vitalidad & $53.1 \pm 20.5$ & $10-95$ \\
\hline
\end{tabular}

DT: Desviación típica

Al comparar la Escala Específica para Enfermedades Renales entre hombres y mujeres, no se encontraron diferencias estadísticamente significativas, tal como puede observarse en la tabla 3.

Tabla 3. Comparación entre hombres y mujeres, de la Escala Específica para Enfermedades Renales.

\begin{tabular}{|lccc}
\hline $\begin{array}{l}\text { Escalas Específicas para } \\
\text { Enfermedades Renales }\end{array}$ & Hombres & Mujeres & Valor de $\mathbf{p}$ \\
\hline Listado de síntomas/problemas & $76.9 \pm 15.9$ & $69.4 \pm 20.8$ & NS \\
\hline Efectos de la enfermedad renal & $66.6 \pm 20.8$ & $65.9 \pm 21.6$ & NS \\
\hline Carga de la enfermedad renal & $57.9 \pm 23.5$ & $45.2 \pm 26.6$ & NS \\
\hline Situación laboral & $39.5 \pm 35.6$ & $23.1 \pm 33$ & NS \\
\hline Función cognitiva & $84 \pm 16.8$ & $83.5 \pm 27.4$ & NS \\
\hline Calidad de las relaciones sociales & $89.2 \pm 15.3$ & $89.7 \pm 17.7$ & NS \\
\hline Función sexual & $45.4 \pm 33.9$ & $59.6 \pm 37.5$ & NS \\
\hline Sueño & $69.5 \pm 17.2$ & $67.5 \pm 23.3$ & NS \\
\hline Apoyo social & $84.2 \pm 23.9$ & $87.2 \pm 22.7$ & NS \\
\hline
\end{tabular}

Cuando compararon las dimensiones del SF-36, entre hombres y mujeres, tampoco se encontraron diferencias significativas, tal como puede apreciarse en la tabla 4.

Tabla 4. Comparación entre hombres y mujeres, en el SF-36..

\begin{tabular}{|lccc|}
\hline Ítems del SF-36 & Hombres & Mujeres & Valor de $\mathbf{p}$ \\
\hline Función física & $60.3 \pm 26$ & $41.5 \pm 32.3$ & NS \\
\hline Rol físico & $35.5 \pm 42.7$ & $32.7 \pm 41.3$ & NS \\
\hline Dolor & $63.4 \pm 28$ & $58.5 \pm 32.1$ & NS \\
\hline Salud general & $42.1 \pm 17.3$ & $35.4 \pm 16.1$ & NS \\
\hline Bienestar emocional & $71.8 \pm 15.7$ & $67.4 \pm 25$ & NS \\
\hline Rol emocional & $80.7 \pm 33.9$ & $48.7 \pm 50.2$ & NS \\
\hline Función social & $80.3 \pm 21.8$ & $85.6 \pm 23.3$ & NS \\
\hline Vitalidad & $53.4 \pm 19.5$ & $52.7 \pm 22.8$ & NS \\
\hline
\end{tabular}

Se encontró una relación estadísticamente significativa entre la edad y la dimensión función sexual $(r=-0.351$, $p<0.05$ ) de la Escala Específica para Enfermedades Renales. De igual forma, se encontró relación estadística entre la edad y las siguientes dimensiones del SF-36: función física $(r=-0.451 ; p<0.01)$, el rol físico $(r=-0.442 ; p<0.05)$ y la vitalidad $(r=-0.422 ; p<0.05)$.

Cuando se relacionó el ICC con cada una de las variables, sólo se encontró correlación estadísticamente significativa con la función física $(r=-0.473 ; p<0.01)$ y la vitalidad $(r=-0.405 ; p<0.05)$, del cuestionario SF-36.

De igual manera, se encontró una correlación estadísticamente significativa entre el ICC y la edad $(r=0.709$; $\mathrm{p}<0.01$ ).

Para tener una referencia respecto a la población general se representaron los valores de las dimensiones del SF-36 de la población estudiada comparada con los valores de la población general española sana para la misma edad (figura 1).

\section{Discusión}

El objetivo del presente estudio fue evaluar la CVRS de los pacientes con ERCA en estadio 5 o etapa pre-diálisis, ya que existe poca bibliografía que documente la calidad de vida de estos pacientes. En efecto, existe bastante bibliografía que analiza la CVRS del paciente con ERCA, pero cuando se encuentran en tratamiento con una TRS; así como comparaciones entre los diferentes procedimientos de TRS ${ }^{12-15}$. 


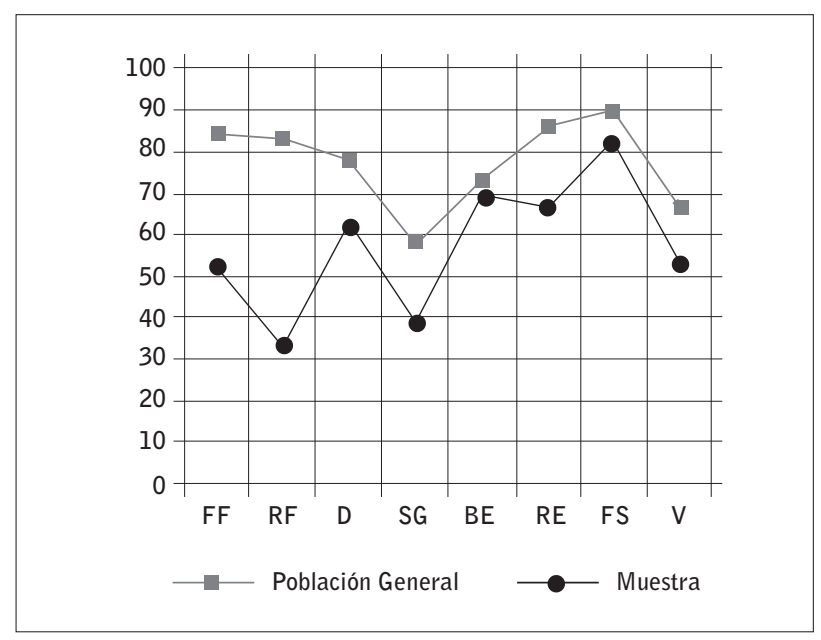

Figura 1. Valores medios de las dimensiones de la población estudiada y la población general española.

Función Física (FF); Rol Físico (RF); Dolor (D); Salud General (SG); Bienestar Emocional (BE); Rol Emocional (RE); Función Social (FS); Vitalidad (V).

En nuestro estudio, decidimos que dentro del grupo de pacientes con ERCA en estadio 5, se evaluaría sólo a aquellos que tenían un $\mathrm{FG}<15 \mathrm{ml} / \mathrm{min}$, puesto que son los que están más próximos a necesitar una TRS y así poder tener alguna referencia. De hecho la edad media de los pacientes que componen la muestra es muy parecida a la de los pacientes en diálisis de nuestro país (16-18). De igual forma, la distribución por género de la muestra se asemeja a la de la población española en diálisis ( $59 \%$ hombres y $41 \%$ mujeres), y similar a 10 publicado por otros autores ${ }^{19-21}$.

También se ha señalado que el aumento de la ERCA y la mayor edad de estos pacientes se acompaña de otras enfermedades asociadas ${ }^{1,22}$ hecho este confirmado en nuestros pacientes, con un ICC elevado (5.81 \pm 2.76$)$, destacando entre las patologías más frecuentes, la diabetes y enfermedades cardiovasculares. Es decir, estamos ante una muestra de pacientes, que aunque no han entrado en diálisis, su perfil es bastante parecido al del paciente que está en diálisis.

Cuando analizamos todas las variables del KDQOL-SF en su conjunto, en las Escalas Específicas para Enfermedades Renales, encontramos como dimensiones más afectadas, la Situación Laboral y la Función Sexual. La Calidad de las Relaciones Sociales, la Función Cognitiva y el Apoyo Social, por el contrario, son las dimensiones mejor conservadas, resultados similares a los publicados en otro estudio ${ }^{23}(89.4 \pm 16.1 ; 83.8 \pm 21.5$ y $85.4 \pm 23.1$ respectivamente). En cuanto a ésta última, varios autores apuntan que el apoyo por parte de seres queridos propicia una mejor calidad de vida ${ }^{24,25}$.
En las dimensiones del cuestionario genérico del SF36, que complementa al KDQOL, el Rol Físico, la Salud General y la Función Física, son las más afectadas, algo común con algún estudio ${ }^{26}$. Por el contrario, la Función Social y el Bienestar Emocional destacan como dimensiones mejor conservadas.

Al separar a hombres y mujeres, en el análisis de las Escalas Específicas para Enfermedades Renales, los hombres puntuaron más alto en la Carga de la Enfermedad Renal y la Situación Laboral y más bajo en la Función Sexual, en comparación con la mujer, pero sin encontrar diferencias estadísticamente significativas entre ambos. En las dimensiones del SF-36, la Función Física y el Rol Emocional, presentaban peor puntuación las mujeres, aunque sin diferencias significativas. Sin embargo, en la mayoría de los estudios sobre CVRS de pacientes en diálisis, la mujer presenta peor calidad de vida, sobre todo en la esfera cognitiva, relacionada con mayor prevalencia de depresión y ansiedad ${ }^{8,27}$. El motivo por el que nuestros resultados no coinciden con estos estudios puede deberse a que la muestra estudiada era pequeña, y tan sólo con 13 mujeres.

Sin embargo, la edad sí parece tener mayor influencia en la CVRS. En efecto, en nuestra muestra encontramos que a mayor edad peor Función Sexual ( $r=-0.351$; $\mathrm{p}<0.05$ ), en la línea de los publicado por otros autores $(23,28-30)$. De igual manera, se encontró que a mayor edad peor Función Física $(r=-0.451 ; p<0.01)$, peor Rol Físico $(r=-0.442 ; p<0.05)$ y peor Vitalidad $(r=-0.422$; $p<0.05)$, similar a lo encontrado en otro estudio ${ }^{31}$.

Por otro lado, se encontró correlación entre el ICC y Función Física ( $r=-0.473 ; p<0.01)$, encontrado también por otros autores ${ }^{26}$ y la Vitalidad $(r=-0.405 ; p<0.05)$; es decir, a mayor comorbilidad disminuyen estas dos dimensiones, que como hemos visto anteriormente están influenciadas también por la edad. Por lo que como es lógico, se encontró una muy buena relación entre la edad y el ICC $(r=0.709 ; p<0.01)$.

Parece evidente, que la variable que más influye en la CVRS, al menos en la muestra analizada, es la edad y su asociación con la comorbilidad. Aunque no hemos encontrado bibliografía específica en la línea de estos resultados, si existen publicaciones que encuentran relación entre "fragilidad", concepto que puede englobar mayor edad y comorbilidad, con peor CVRS. Varios estudios muestran que a mayor "fragilidad" peores puntuaciones en las dimensiones físicas y mental, aunque no en la social ${ }^{32,33}$. 
Cuando se compararon las dimensiones del cuestionario genérico SF-36 de la muestra con la población sana de referencia (edad), observamos que excepto en la dimensión Bienestar Emocional, en el resto de dimensiones las puntuaciones son sensiblemente más bajas, similar a lo comunicado en los pacientes en hemodiálisis ${ }^{16,18}$.

Siendo conscientes de la influencia que ejerce la ERCA en la CVRS, sería conveniente que los pacientes acudieran precozmente a los servicios de nefrología y, se pusieran a disposición del equipo multidisciplinar que lo forma; ya que en esta etapa de la enfermedad es crucial el control de todas las variables que intervienen en la misma ${ }^{8,22,34,35}$ y así poder prevenir complicaciones y retrasar la afectación en la calidad de vida que esta enfermedad condiciona.

La principal limitación de este estudio ha sido la muestra, debido a que en estadio 5 de la ERCA y con GF $<15 \mathrm{ml} / \mathrm{min}$, no hay muchos pacientes en la UGC de Nefrología de Córdoba y para recoger una muestra más amplia habría que haber alargado el tiempo de duración del estudio. Otra limitación, ha sido la poca bibliografía que analice la CVRS en esta etapa de la ERCA, pues en algunos casos hemos utilizado estudios de pacientes en diálisis para poder compararlos con nuestros resultados al no disponer de estudios que analizaran la calidad de vida en esta etapa de la enfermedad.

A la vista de nuestros resultados podemos concluir que al menos, en la muestra estudiada, los pacientes con ERCA en estadio 5 con un FG bajo tienen disminuida su CVRS con afectación en la mayoría de las dimensiones que engloba este concepto; en comparación con la población sana de referencia y muy similar, y parecida a la de los pacientes en diálisis.

Las dimensiones de la CVRS más afectadas en esta etapa de la ERCA son la Carga de la Enfermedad Renal, la Situación Laboral, la Función Sexual, Rol Físico, Función Física, Salud General y Vitalidad.

Las variables más influyentes en la disminución de la CVRS son la edad y la comorbilidad, no habiéndose encontrado relación con respecto al sexo.

Para finalizar, y dada la escasez de bibliografía sobre el estudio de la CVRS en esta etapa de la ERCA, sería conveniente y recomendable, que se llevaran a cabo nuevas investigaciones, para así poder establecer más referencias científicas al respecto.
Recibido: 8 junio 2017

Revisado: 21 junio 2017

Modificado: 30 junio 2017

Aceptado: 5 agosto 2017

\section{Bibliografía}

1. Otero A, de Francisco A, Gayoso P, García F. Prevalencia de la insuficiencia renal crónica en España: Resultados del estudio EPIRCE. Enferm Nefrología. 2010; 30:78-86. DOI: 10.3265/ Nefrologia.pre2009.Dic.57322.

2. Levey AS, Coresh J. Chronic kidney disease. The Lancet. 2012; 379 (9811):165-80. D0I: 10.1016/ S0140-6736(11)60178-5.

3. Gutiérrez Sánchez D, Leiva-Santos JP, SánchezHernández R, Gómez García R. Prevalencia y evaluación de síntomas en enfermedad renal crónica avanzada. Enferm Nefrológica. 2015; 18(3):22836. D0I: $10.4321 / S 2254-28842015000300010$.

4. Registro de Diálisis y Trasplante. Sociedad Española de Nefrología. [Consultado 5 junio 2017]. Disponible en: http://www.senefro.org/modules.php?name $=$ webstructure \&idwebstructure $=29$.

5. Brown MA, Collett GK, Josland EA, Foote $\mathrm{C}, \mathrm{Li}$ Q, Brennan FP. CKD in Elderly Patients Managed without Dialysis: Survival, Symptoms, and Quality of Life. Clin J Am Soc Nephrol. 2015; 10(2):2608. D0I: 10.2215/CJN.03330414.

6. Kaltsouda A, Skapinakis $P$, Damigos D, Ikonomou $M$, Kalaitzidis R, Mavreas V, et al. Defensive coping and health-related quality of life in chronic kidney disease: a cross-sectional study. BMC Nephrol. 2011; 12:28. D0I: 10.1186/1471-2369-12-28.

7. Rebollo Rubio A, Morales-Asencio JM, PonsRaventos ME, Mansilla-Francisco JJ. Revisión de estudios sobre calidad de vida relacionada con la salud en la enfermedad renal crónica avanzada en España. Nefrología. 2015; 35:92-109. D0I: 10.3265/Nefrologia.pre2014.Jul.12133. 
8. Jofre R. Factores que afectan a la calidad de vida en pacientes en prediálisis, diálisis y trasplante renal. Nefrología. 1999; 19 (1):84-90.

9. Seguí Gomà A, Amador Peris P, Alcario R, Belén A. Calidad de vida en pacientes con insuficiencia renal crónica en tratamiento con diálisis. Rev Soc Esp Enferm Nefrol 2010; 13(3):155-60.

10. Álvarez-Ude F, Vicente E, Badía X. La medida de la calidad de vida relacionada con la salud en los pacientes en programa de hemodialisis y dialisis peritoneal continua ambulatoria de Segovia. Enferm Nefrol 1995; 15:572-80.

11. Ruiz Román M J, Román Cereto M, Martín Reyes G, Alférez Alférez M J, Prieto Merino D. Calidad de Vida Relacionada con la Salud en las diferentes terapias sustitutivas de la Insuficiencia Renal Crónica. Rev Soc Esp Enferm Nefrol. 2003; 6 (4): 6-16.

12. Álvares J, Cesar CC, de Assis Acurcio F, Andrade EI, Gurgel, Cherchiglia ML. Quality of life of patients in renal replacement therapy in Brazil: comparison of treatment modalities. Qual Life Res Dordr. 2012; 21(6):983-91. D0I: 10.1007/s11136-011-0013-6.

13. Czyzewski L, Sanko-Resmer J, Wyzgał J, Kurowski A. Assessment of health-related quality of life of patients after kidney transplantation in comparison with hemodialysis and peritoneal dialysis. Ann Transplant. 2014; 19:576-85. D0I: 10.12659/ A0T.891265.

14. Tannor EK, Archer E, Kapembwa K, van Schalkwyk SC, Davids MR. Quality of life in patients on chronic dialysis in South Africa: a comparative mixed methods study. BMC Nephrol Lond. 2017; 18:4. DOI: 10.1186/s12882-016-0425-1.

15. Adell Lleixà $M$, Casadó Marin $L$, Andújar Solé J, Solà Miravete E, Martínez Segura E, Salvadó Usach T. Valoración de la calidad de vida relacionada con la salud en la enfermedad renal crónica terminal mediante un cuestionario de resultados percibidos por los pacientes. Enferm Nefrol 2016; 19(4):33140. D0I: $10.4321 / S 2254-28842016000400004$.

16. González L A, Nava R Á, Andrés M. B, Herrera G F, Santana Z H, Diego M J, et al. Grado de adherencia y conocimiento previo a la conciliación terapéutica en pacientes en diálisis peritoneal.
Enferm Nefrol 2016; 36:459-60. D0I: 10.1016/j. nefro.2015.10.021.

17. Frías Albañil T, Ramírez Moreno MC, Crespo Montero R. Análisis de la calidad de vida en pacientes eh hemodiálisis ambulatoria y su relación con el nivel de dependencia. Enferm Nefrol. 2014; 17(3):167-74

18. Ahís Tomás $P$, Peris Ambou IC, Mneu Oset M, Perez Baylach CM, Bonilla Culebras B, Panizo González N. Impacto subjetivo de las estrategias no farmacológicas de mejora de la calidad de vida y el cumplimiento terapéutico en pacientes en hemodiálisis. Rev Soc Esp Enferm Nefrol. 2017; 20(1):22-7. DOI: $10.4321 / S 2254-28842016000400004$.

19. Martín F, Reig A, Sarró F, Ferrer R, Arenas D, González F, Gil T. Evaluación de la calidad de vida en pacientes de una unidad de hemodiálisis con el cuestionario Kidney Disease Quality of Life - Short Form (KDQOL-SF). DYT. 2004; 25 (2): 79-92

20. Arruda Costa GM, Gomes Pinheiro MB, Medeiros Soraya $M{ }^{a}$, Rainere de Oliveira Costa E, Santos Cossi M. Calidad de vida en pacientes con insuficiencia renal crónica en hemodiálisis, Enferm Global. 2016; 43:59-71.

21. Rebollo Rubio A, Asensio M, Miguel J, Raventos $P$, Eugenia $M$ a. Influencia de la consulta de enfermería de enfermedad renal crónica avanzada en pacientes que inician tratamiento renal sustitutivo. Enferm Nefrol. 2014; 17(4):243-50. D0I: $10.4321 / S 2254-28842014000400002$.

22. Poersch RF, Andrade FP, Bosco AD, Edit Rovedder PM. Quality of life of chronic renal failure patients undergoing hemodialysis. ConScientiae Saúde Sao Paulo. 2015; 14(4):608-16.

23. Ibrahim N, Teo SS, Din NC, Gafor AH, Ismail R. The Role of Personality and Social Support in HealthRelated Quality of Life in Chronic Kidney Disease Patients. PLoS One. 2015; 10(7). D0I: 10.1371/ journal.pone.0129015.

24. Kara B, Caglar K, Kilic S. Nonadherence with diet and fluid restrictions and perceived social support in patients receiving hemodialysis. J Nurs Scholarsh Off Publ Sigma Theta Tau Int Honor Soc Nurs. 2007; 39(3):243-8. D0I: 10.111l/j.15475069.2007.00175.x. 
25. Pagels AA, Söderkvist BK, Medin C, Hylander B, Heiwe $S$. Health-related quality of life in different stages of chronic kidney disease and at initiation of dialysis treatment. Health Qual Life Outcomes. 2012; 10:71. DOI: 10.1186/1477-7525-10-71.

26. Cruz MC, Andrade C, Urrutia M, Draibe $S$, Nogueira-Martins LA, de Castro Cintra Sesso R. Quality of life in patients with chronic kidney disease. Clinics. 2011; 66(6):991-5. D0I: 10.1590/ S1807-59322011000600012.

27. Peng Y-S, Chiang C-K, Kao T-W, Hung K-Y, Lu C-S, Chiang S-S, et al. Sexual dysfunction in female hemodialysis patients: a multicenter study. Kidney Int. 2005; 68(2):760-5. DOI: 10.1111/j.15231755.2005.00454.x

28. Martín-Díaz F, Reig-Ferrer A, Ferrer-Cascales R. [Sexual function and quality of life in hemodialysis male patients]. Nefrol Publicacion Of Soc Espanola Nefrol. 2006; 26(4):452-60.

29. Azevedo P, Santos R, Duraes J, Santos 0, Carvalho MJ, Cabrita $A$, et al. Disfunción sexual en pacientes en diálisis peritoneal: relación con factores metabólicos y con la percepción de la calidad de vida. Enferm Nefrol. 2014; 34:703-9. DOI: 10.3265/Nefrologia.pre2014.Jul.12548.

30. Santos PR. Relationship between gender and age with quality of life in chronic hemodialysis patients. Rev Assoc Médica Bras. 2006; 52(5):356-9. D0I: $10.1590 /$ S0104-42302006000500026.

31. Mansur HN, Colugnati FA, Grincenkov FR dos $S$, Bastos MG. Frailty and quality of life: a crosssectional study of Brazilian patients with predialysis chronic kidney disease. Health Qual Life Outcomes. 2014; 12:27. DOI: 10.1186/14777525-12-27.
32. Lee SJ, Son $H$, Shin SK. Influence of frailty on health-related quality of life in pre-dialysis patients with chronic kidney disease in Korea: a crosssectional study. Health Qual Life Outcomes. 2015; 13:70. DOI: 10.1186/s12955-015-0270-0.

33. Sakhuja A, Hyland J, Simon JF. Managing advanced chronic kidney disease: a primary care guide. Cleve Clin J Med. 2014; 81(5):289-99. D0I: 10.3949/ ccjm.81a.13046.

34. Boini S, Frimat L, Kessler M, Briançon S, Thilly N. Predialysis therapeutic care and healthrelated quality of life at dialysis onset (The pharmacoepidemiologic AVENIR study). Health Qual Life Outcomes. 2011; 9:7.

35. Forero Villalobos J, Barrios Araya S. Rol de enfermería en la consulta de prediálisis en el paciente con enfermedad renal crónica avanzada. Enferm Nefrol. 2016; 19(1):77-86. 